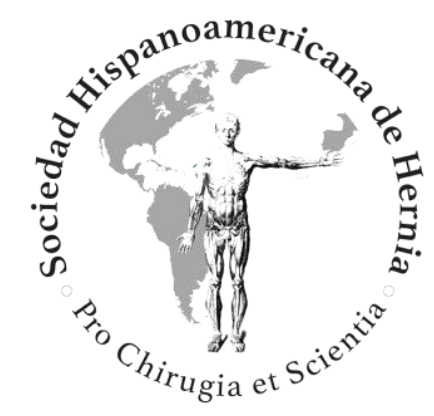

Plastia inguinal laparoscópica tipo TAPP asociada a resección intestinal como tratamiento en hernia de Richter

\title{
TAPP laparoscopic inguinal hernia repair associated with intestinal resection to solve a Richter's hernia
}




\section{371}

\section{Plastia inguinal laparoscópica tipo TAPP asociada a resección intestinal como tratamiento en hernia de Richter}

TAPP laparoscopic inguinal hernia repair associated with intestinal resection to solve a Richter's hernia

Ana Paula Ruiz Funes Molina, Jorge Meza Carmona, Jorge Adrián Romero Sánchez, Luis Roberto García Arrona

Cirugía General. Hospital Central Sur de Alta Especialidad. PEMEX. Ciudad de México (México)

Recibido: $28 / 08 / 2020$

Aceptado: $28 / 10 / 2020$

Autor para correspondencia: Ana Paula Ruiz Funes Molina. Cirugía General. Hospital Central Sur de Alta Especialidad. PEMEX. Anillo Perif. 4091, Fuentes del Pedregal. Tlalpan, 14140. Ciudad de México (México)

Correo electrónico: draruizfunesmolina@gmail.com

DOI: 10.20960/rhh.00371

Conflicto de interés: los autores declaran no tener conflicto de intereses.

\section{RESUMEN}

Introducción: La hernia de Richter es un tipo de hernia particular donde únicamente se estrangula una porción de la circunferencia del intestino, debido a que el borde antimesentérico se incarcera dentro del defecto herniario. Requiere manejo quirúrgico urgente y puede ser tratada mediante cirugía laparoscópica. 
Caso clínico: Presentamos caso de paciente femenino de 77 años que acudió al Servicio de Urgencias con cuadro de dolor abdominal y oclusión intestinal. En región inguinal izquierda se evidenció tumor palpable de 3 × 3 $\mathrm{cm}$, no reductible, doloroso a la palpación, compatible con una hernia inguinal indirecta. En los exámenes de laboratorio destacó la presencia de leucocitosis y lactato de 3.2. Se realizó tomografía abdominopélvica con hallazgos compatibles con hernia incarcerada. De acuerdo con los hallazgos previamente mencionados, se decidió programación para laparoscopia diagnóstica con intención de plastia inguinal tipo TAPP. Se encontró una hernia inguinal izquierda recidivante con contenido de borde antimesentérico de íleon (hernia de Richter) y fibrosis por malla previa. Se realizó plastia inguinal izquierda tipo TAPP, asociada a minilaparotomía con resección y entero-entero anastomosis. La paciente cursó con adecuada evolución posquirúrgica.

Discusión: La realización de una plastia inguinal laparoscópica tiene beneficios para un paciente con hernia complicada y es útil para el diagnóstico y tratamiento de hernias menos frecuentes, como la hernia de Richter.

Palabras clave: Hernia inguinal, hernia de Richter, laparoscopia, obstrucción intestinal.

\section{ABSTRACT}

Background: Richter's hernia is a particular type of hernia where only a portion of the circumference of the intestine is strangled, due to the antimesenteric border being incarcerated within a hernial ring. This disease requires urgent surgical management and may be treated by laparoscopic surgery.

Case report: We present the case of a 77-year-old female patient who came to the emergency department with abdominal pain and symptoms of intestinal obstruction. The left inguinal region revealed a palpable tumor 
measuring $3 \times 3 \mathrm{~cm}$, non-reducible, painful upon palpation, compatible with an indirect inguinal hernia. In the laboratory tests, the presence of leukocytosis and lactate of 3.2 stood out. Abdominopelvic tomography was performed with findings consistent with an incarcerated hernia. Based on the previously mentioned findings, it was decided to schedule a diagnostic laparoscopy with the intention of TAPP inguinal repair. A recurrent left inguinal hernia with the content of the ileum antimesenteric border (Richter's hernia) and fibrosis due to previous mesh was found. A TAPP left inguinal repair was performed, associated with minilaparotomy with resection and enteroenterostomy. The patient had an adequate postoperative evolution.

Discussion: Laparoscopic inguinal hernia repair is beneficial for a patient with a complicated hernia and is considered useful for diagnosis and treatment for less frequent hernias, such as a Richter's hernia.

Keywords: Inguinal hernia, Richter's hernia, laparoscopy, intestinal obstruction.

\section{INTRODUCCIÓN}

El manejo quirúrgico de las hernias inguinales ha evolucionado considerablemente con el paso del tiempo. Actualmente existen dos técnicas endoscópicas para la reparación de hernias inguinales, transabdominal preperitoneal (TAPP) y totalmente extraperitoneal (TEP) ${ }^{1}$. Su aplicación generalmente se limita a procedimientos programados $o$ hernias no complicadas, sin embargo, se han descrito ventajas en procedimientos urgentes, como la capacidad para visualizar el órgano incarcerado o estrangulado directamente y la posibilidad de realizar la resección laparoscópica².

La hernia de Richter es un tipo de hernia particular donde únicamente se estrangula una porción de la circunferencia del intestino debido a que el borde antimesentérico se incarcera dentro del defecto herniario. Es una 
entidad poco frecuente y representa únicamente el $1 \%$ de las hernias de pared abdominal; generalmente se diagnostica cuando se asocia a una obstrucción intestinal ${ }^{3}$. Se requiere de una adecuada sospecha diagnóstica asociada a un tratamiento oportuno para evitar complicaciones mayores ${ }^{4}$.

No está estandarizado el abordaje de elección en este tipo de hernias, por lo que la selección del abordaje quirúrgico depende de la etapa del cuadro clínico con la que se presenta el paciente, la naturaleza de la hernia, el riesgo relacionado con cada una de las técnicas quirúrgicas, las destrezas del cirujano y la experiencia del equipo médico ${ }^{5}$.

\section{CASO CLÍNICO}

Mujer de 77 años, con antecedente de hipertensión arterial sistémica, plastia inguinal izquierda abierta hace 14 años con colocación de material protésico, con abordaje anterior; sin otros antecedentes relevantes. Es referida al servicio de Cirugía General por presentar dolor abdominal y vómito de contenido biliar de 24 horas de evolución.

A la exploración física se encontró con tensión arterial dentro de los parámetros normales, con frecuencia cardiaca de 100 latidos por minuto, con abdomen doloroso a la palpación, sin datos de irritación peritoneal. A la auscultación se escuchó peristalsis aumentada. En región inguinal izquierda se evidenció tumor palpable de $3 \times 3 \mathrm{~cm}$, no reductible, doloroso a la palpación, compatible con una hernia inguinal indirecta. En los exámenes de laboratorio destacó la presencia de leucocitosis y lactato de 3.2.

En la tomografía abdominopélvica (fig. 1) se observó tumor de $3.2 \times 2 \times 8 \mathrm{~cm}$ en región inguinal izquierda compatible con hernia incarcerada.

De acuerdo con los hallazgos previamente mencionados se decidió programación para laparoscopia diagnóstica con intención de plastia inguinal tipo TAPP. Se administró una dosis profiláctica de antibiótico (cefalosporina de tercera generación).

Una vez con la paciente en posición y bajo anestesia general, se realizó colocación de trocar de $12 \mathrm{~mm}$ con técnica de Hasson, umbilical, y 
posteriormente se hizo laparoscopia diagnóstica. Se encontró una hernia inguinal izquierda recidivante con contenido de borde antimesentérico de íleon (hernia de Richter) y fibrosis por malla previa. Se colocaron dos trocares accesorios de $5 \mathrm{~mm}$ en ambos flancos. Se realizó reducción de saco herniario con necrosis de segmento intestinal, sin evidencia de perforación. Se identificó un defecto indirecto de $2 \mathrm{~cm}$. Se inició disección de colgajo peritoneal para continuar con plastia inguinal con técnica transabdominal preperitoneal con colocación de malla (fig. 2). Se colocó malla de polipropileno medianamente pesada de $15 \times 12 \mathrm{~cm}$. Se fijó malla con fijadores absorbibles a ligamento de Cooper, recto abdominal y pared lateral del abdomen; se evidenció adecuada colocación de la misma. Se cerró peritoneo con surgete continuo con sutura barbada monofilamento de absorción lenta.

Una vez terminada la plastia, se realizó ampliación del puerto umbilical para exponer el segmento intestinal comprometido, el cual era de $5 \mathrm{~cm}$. Se decidió resección intestinal extracorpórea con anastomosis latero-lateral mecánica con engrapadora lineal $75 \mathrm{~mm}$ (fig. 3). Se concluyó el procedimiento sin complicaciones asociadas.

La paciente presentó adecuada evolución en el posoperatorio inmediato y mediato; se inició vía oral a las 48 horas, con adecuada tolerancia. No hubo evidencia de complicaciones tempranas asociadas.

Fue egresada al quinto día del posoperatorio y no ha mostrado complicaciones en los 18 meses de seguimiento.

\section{DISCUSIÓN}

La hernia de Richter se produce cuando la porción antimesentérica del intestino se estrangula dentro de un anillo herniario. Generalmente es unilateral y puede aparecer en cualquier localización de la pared abdominal, pero es más frecuente en la región inguinal y femoral. Por su baja frecuencia generalmente se diagnostica en presencia de obstrucción intestinal o complicaciones graves como la perforación intestinal ${ }^{3}$. Debido a lo anterior, 
se requiere de una adecuada sospecha diagnóstica asociada a un tratamiento oportuno para evitar complicaciones mayores ${ }^{4}$.

Entre los factores que se asocian a complicaciones posoperatorias y elevada mortalidad se encuentran la presencia de hernia por tiempo prolongado y la irreductibilidad, el retraso en la admisión hospitalaria y la presencia de comorbilidades ${ }^{4}$.

La decisión de realizar una cirugía abierta versus laparoscópica depende del grado de experiencia del equipo quirúrgico y las habilidades del cirujano, el riesgo acorde a la técnica quirúrgica en cada paciente, la naturaleza de la hernia y la presentación clínica del paciente ${ }^{5}$.

El abordaje por medio de cirugía de mínimo acceso, con plastia inguinal tipo transabdominal preperitoneal (TAPP) o totalmente extraperitoneal (TEP) puede ser considerado como una opción terapéutica adecuada para el tratamiento de hernias incarceradas, siempre que se haga una selección apropiada de paciente ${ }^{6,7}$.

Se describen dificultades técnicas asociadas a la reducción del saco herniario y su contenido al abdomen, así como un incremento en el riesgo de lesión a otros órganos durante el procedimiento. Por otro lado, la visualización directa del órgano incarcerado o estrangulado y la posibilidad de una resección intestinal laparoscópica o extracorpórea, asociada a la plastia inguinal, ofrecen ventajas significativas ${ }^{4,8}$.

En un análisis retrospectivo realizado por Yang, con un total de 188 casos, se compararon casos de hernias incarceradas en tratamiento laparoscópico y abierto. Se encontró una menor tasa de infección en cirugía laparoscópica, así como una disminución en el tiempo de estancia hospitalaria ${ }^{9}$. Esto muestra un beneficio cuando se operan cirugías por medio de cirugía de mínimo acceso.

El riesgo asociado a la infección de la malla confiere cierta incertidumbre, sin embargo, no colocar malla se asocia a un incremento en el riesgo de recidiva. Hay autores que recomiendan la hernioplastia con malla en un segundo evento quirúrgico ${ }^{10}$, pero se ha observado que no hay 
contraindicación para colocación de malla asociada a una resección intestinal ${ }^{11}$. Generalmente se prefieren mallas sintéticas con adecuada fijación, sin olvidar que, en presencia de peritonitis, su uso está en controversia $^{12}$.

Entre los principales beneficios del abordaje laparoscópico se encuentra una mayor capacidad diagnóstica. Permite la detección de hernias bilaterales con la capacidad de hacer una plastia de ambas regiones inguinales en el mismo tiempo quirúrgico y tiene superioridad en la reparación de hernias de piso pélvico como hernia obturatriz. Asociado a lo anterior, el abordaje de mínimo acceso disminuye la tasa de laparotomías y reduce la tasa de infección ${ }^{6}$, con lo que disminuyen el tiempo de recuperación y las complicaciones asociadas.

\section{CONCLUSIÓN}

La realización de una plastia inguinal laparoscópica tiene beneficios para un paciente con hernia complicada y es útil para el diagnóstico y tratamiento de hernias menos frecuentes, como la hernia de Richter. El abordaje por medio de cirugía de mínima invasión permite el beneficio de una plastia laparoscópica asociada a una incisión menor para la resección intestinal o extracción del segmento intestinal comprometido. La decisión de la colocación de una malla asociada a una cirugía con resección intestinal depende del estado de los tejidos locales y la contaminación durante la cirugía. Es importante destacar que cada caso debe ser individualizado.

\section{BIBLIOGRAFÍA}

1. The HerniaSurger Group. International guidelines for groin hernia management. Hernia. 2018;22:1-165. DOI: 10.1007/s10029-017-1668$x$

2. Gómez Menchero J, Suárez Grau JM, Gila Bohórquez A, García Moreno J, Landra P, Ferrufino J, et al. Reparación laparoscópica de emergencia de una hernia inguinal intersticial estrangulada, ¿es factible? Cir Andal. 2019;30(1):153-4. 
3. Rodríguez Hermosa JI, Tuca Rodríguez F, Codina Cazador A, Gironès Vilà J, Lequerica Cabello MA, Roig García J, et al. Hernia de Richter como causa de obstrucción del intestino delgado. Cir Esp. 2001;69(1):65-7. DOI: 10.1016/S0009-739X(01)71688-0

4. Kafadar MT, Gök MA. Laparoscopic transabdominal preperitoneal repair of strangulated femoral hernia: Superiority of an unusual emergency surgical approach due to a case. Ann Med Surg (Lond). 2018;36:110-2. DOI: 10.1016/j.amsu.2018.10.014

5. Jagad RB, Shah JR, Patel G. The laparoscopic transperitoneal approach for irreducible inguinal hernias: Perioperative outcome in four patients. J Minim Access Surg. 2009;5(2):31-4. DOI: 10.4103/0972-9941.55104

6. Yang GPC. Laparoscopy in emergency hernia repair. Ann Laparosc Endosc Surg. 2017;2:107-107. DOI: 10.21037/ales.2017.05.05

7. Rebuffat C, Galli A, Scalambra MS, Balsamo F. Laparoscopic repair of strangulated hernias. Surg Endosc Other Interv Tech. 2006;20(1):131-4. DOI: 10.1007/s00464-005-0171-0

8. Cox TC, Huntington CR, Blair LJ, Prasad T, Heniford BT, Augenstein VA. Quality of life and outcomes for femoral hernia repair: does laparoscopy have an advantage? Hernia. 2017;21(1):79-88.

9. Yang GP, Chan CT, Lai EC, Chan OC, Tang CN, Li MK. Laparoscopic versus open repair for strangulated groin hernias: 188 cases over 4 years. Asian J Endosc Surg. 2012;5(3):131-7. DOI: 10.1111/j.17585910.2012.00138.x

10. Hoffman A, Leshem E, Zmora O, Nachtomi O, Shabtai M, Ayalon $A$, et al. The combined laparoscopic approach for the treatment of incarcerated inguinal hernia. Surg Endosc. 2010;24(8):1815-8. DOI: 10.1007/s00464-009-0857-9

11. Abd Ellatif ME, Negm A, Elmorsy G Al-Katary M, Yousef Ael-A, Ellaithy R. Feasibility of mesh repair for strangulated abdominal wall hernias. Int J Surg. 2012;10:153-6. DOI: 10.1016/j.ijsu.2012.02.004

12. Ospina DA, Toscano CAO, Arango NF, Leguia MKF. Hernia obturatriz y obstrucción intestinal. Reporte de caso. Rev Hispanoam Hernia. 2016;4(4):179-83. 

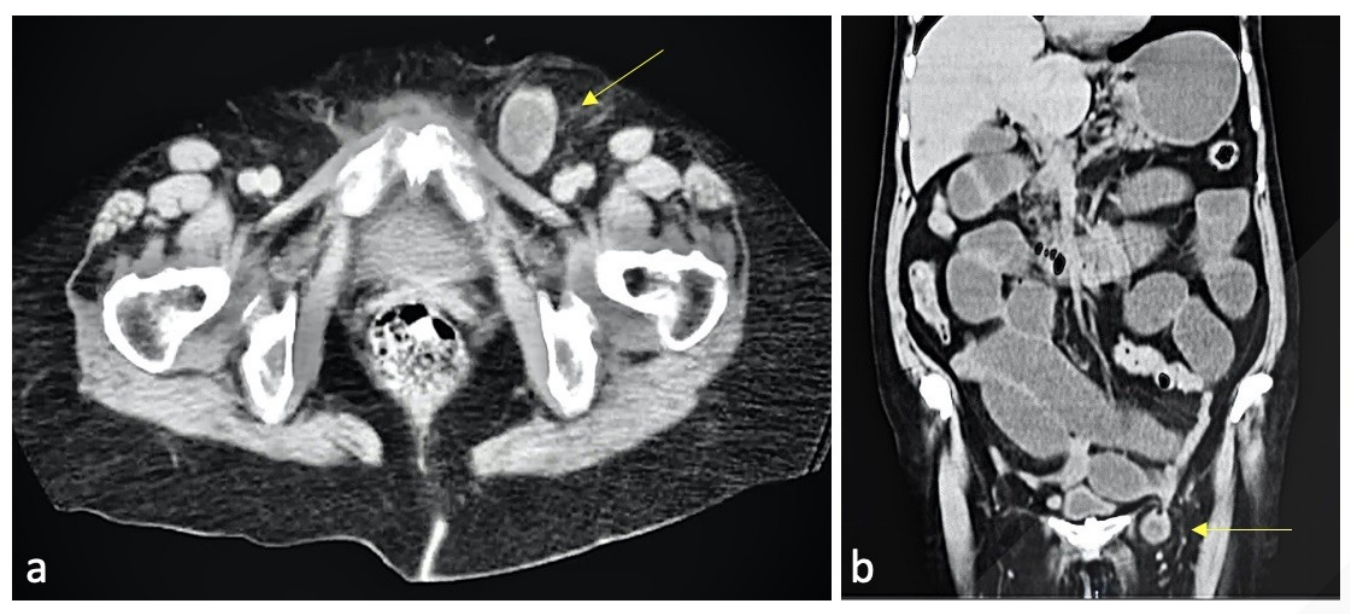

Fig.1. Tomografía abdominopélvica. A. Corte axial con evidencia de hernia inguinal izquierda incarcerada (señalado por flecha). B. Corte coronal con evidencia de hernia inguinal izquierda incarcerada (señalado por flecha).
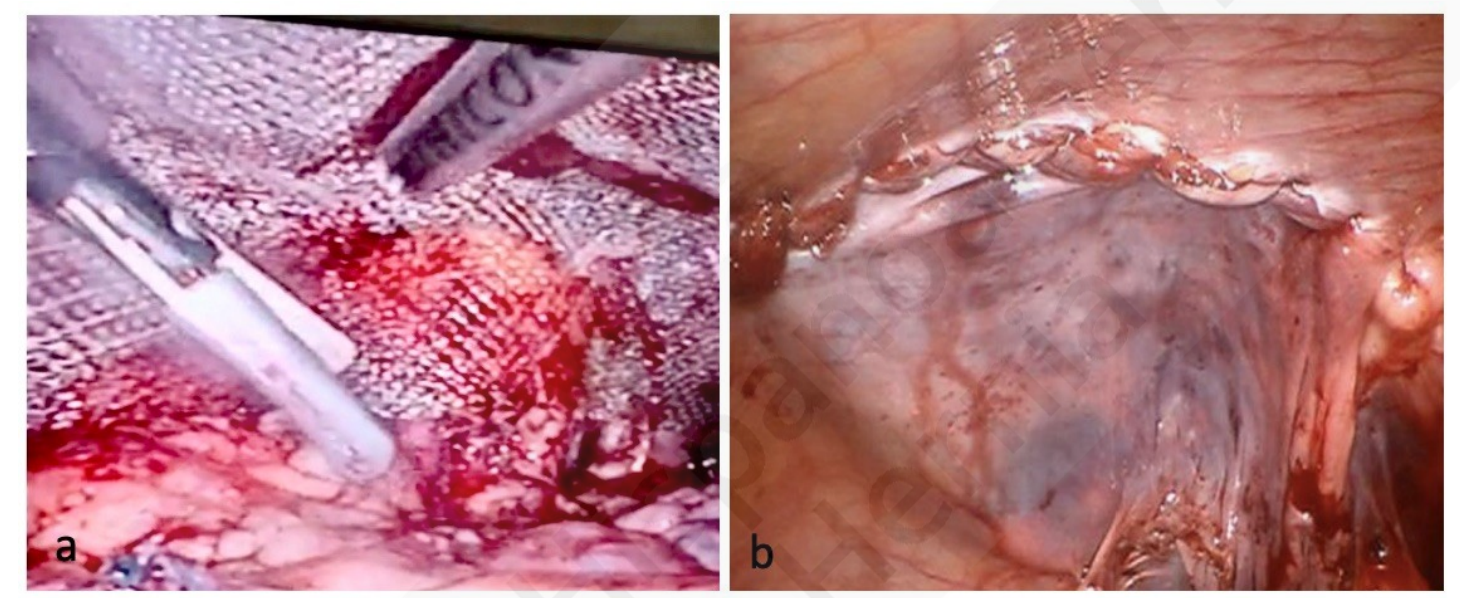

Fig. 2. Plastia laparoscópica transabdominal preperitoneal. A. Colocación de malla preperitoneal. B. Cierre de flap peritoneal. 

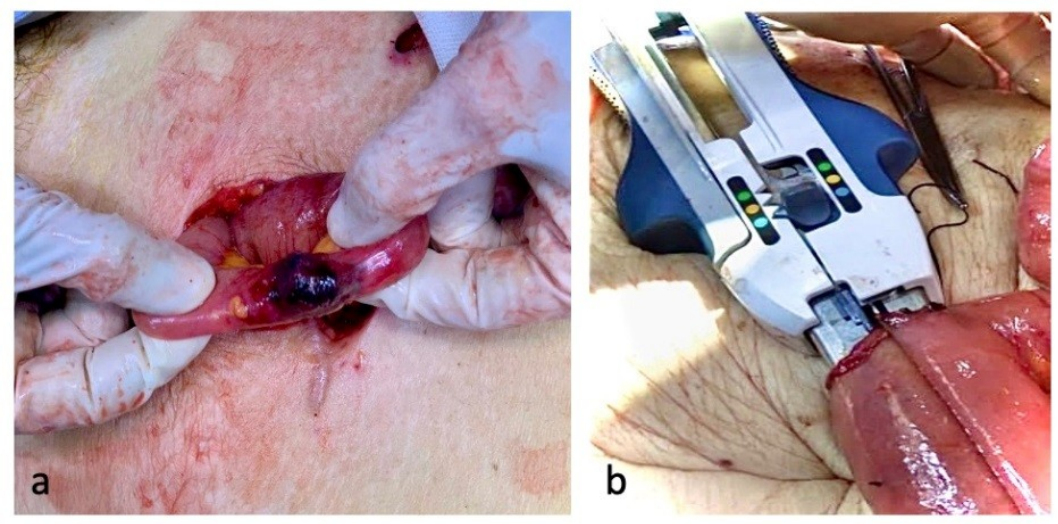

Fig. 3. Resección intestinal. A. Exteriorización de segmento intestinal comprometido con evidencia de necrosis. B. Entero-entero anastomosis latero-lateral mecánica extracorpórea. 